Check for updates

Cite this: Chem. Commun., 2017, 53,5380

Received 23rd March 2017, Accepted 19th April 2017

DOI: $10.1039 / c 7 c c 02208 e$

rsc.li/chemcomm

\section{Two cations, two mechanisms: interactions of sodium and calcium with zwitterionic lipid membranes $\dagger$}

\author{
Matti Javanainen, (D) $\ddagger^{\mathrm{ab}}$ Adéla Melcrová, $\ddagger^{\mathrm{c}}$ Aniket Magarkar, (D) de Piotr Jurkiewicz, (D) *c \\ Martin Hof, (D) C Pavel Jungwirth (D)*da and Hector Martinez-Seara (D) *da
}

\begin{abstract}
Adsorption of metal cations onto a cellular membrane changes its properties, such as interactions with charged moieties or the propensity for membrane fusion. It is, however, unclear whether cells can regulate ion adsorption and the related functions via locally adjusting their membrane composition. We employed fluorescence techniques and computer simulations to determine how the presence of cholesterol-a key molecule inducing membrane heterogeneity-affects the adsorption of sodium and calcium onto zwitterionic phosphatidylcholine bilayers. We found that the transient adsorption of sodium is dependent on the number of phosphatidylcholine head groups, while the strong surface binding of calcium is determined by the available surface area of the membrane. Cholesterol thus does not affect sodium adsorption and only plays an indirect role in modulating the adsorption of calcium by increasing the total surface area of the membrane. These observations also indicate how lateral lipid heterogeneity can regulate various ion-induced processes including adsorption of peripheral proteins, nanoparticles, and other molecules onto membranes.
\end{abstract}

Metal cations play a central role in cell function and regulation. ${ }^{1,2}$ In resting mammalian cells, the most common monovalent cations $\mathrm{Na}^{+}$and $\mathrm{K}^{+}$(at concentrations of 100-150 mM) are segregated between the extracellular and cytosolic compartments,

\footnotetext{
${ }^{a}$ Laboratory of Physics, Tampere University of Technology, P. O. Box 692, FI-33101 Tampere, Finland

${ }^{b}$ Department of Physics, University of Helsinki, P. O. Box 64, FI-00014 University of Helsinki, Finland

${ }^{c} J$. Heyrovský Institute of Physical Chemistry, Czech Academy of Sciences, Dolejskova 3, 18223 Prague 8, Czech Republic.

E-mail: piotr.jurkiewicz@jhinst.cas.cz

${ }^{d}$ Institute of Organic Chemistry and Biochemistry, Czech Academy of Sciences, Flemingovo nam. 2, 16610 Prague 6, Czech Republic.

E-mail: pavel.jungwirth@uochb.cas.cz, hseara@gmail.com

${ }^{e}$ Faculty of Pharmacy, University of Helsinki, Viikinkaari 5E,

FI-00014 University of Helsinki, Finland

$\dagger$ Electronic supplementary information (ESI) available: Table of all simulated systems. Detailed descriptions of system setup, simulation, and analyses. Additional results. See DOI: $10.1039 / \mathrm{c} 7 \mathrm{cc} 02208 \mathrm{e}$

\# These authors contributed equally to this work.
}

respectively. Free divalent cations are more scarce. For example, $\mathrm{Ca}^{2+}$ exhibits a significantly lower concentration on the cytosolic side (1-100 nM) than on the extracellular one (1-2 mM). ${ }^{3}$ Global changes in the distribution of these cations due to cell homeostasis trigger changes in the cell volume, osmotic balance regulation, and electrostatic potential. ${ }^{1,2}$ For these reasons, concentrations of cations and their fluxes in and out from the cell are tightly regulated. ${ }^{1}$ More localized changes of the cation distributions play an important role in, e.g., membrane fusion ${ }^{4,5}$ and signaling., Furthermore, the differential accumulation of cations at the surface of a membrane can ultimately modulate its interaction with the surrounding macromolecules or locally alter the physical properties of the membrane..$^{8-10}$

Over the past decades, considerable progress has been made in unraveling the role of ions in signaling ${ }^{6}$ as well as the regulation of cell volume ${ }^{1}$ and transmembrane potential. ${ }^{2}$ However, it is only recently that advances have been made in the experimental description of the direct interactions between ions and membranes, ${ }^{11,12}$ the quantitative aspects of which are still highly controversial. ${ }^{13}$ Moreover, it remains to be determined whether cells possess mechanisms to selectively modulate their membrane affinity for certain ions.

There is clear evidence that ions interact in a specific way with membranes. ${ }^{12,13}$ While monovalent alkali cations do not adsorb efficiently to membranes unless negatively charged lipids are present, ${ }^{11}$ divalent ions such as $\mathrm{Ca}^{2+}$ do so more readily even if no attractive net negative charge is present. ${ }^{12}$ The extracellular leaflet of the cell membrane is mainly composed of zwitterionic phosphatidylcholine and sphingomyelin together with cholesterol, all of which are overall neutral lipids. Therefore, any selective adsorption of cations onto the surface of such a membrane results in a positive net charge at the membranewater interface. ${ }^{14,15}$ Cells can very precisely control and regulate membrane lipid compositions both globally and locally. ${ }^{16}$ If such changes in composition could also alter membrane-cation interactions, the cell would possess a powerful tool to induce and control a plethora of functions. As the outer layer of the plasma membrane is mostly constituted by zwitterionic and neutral lipids, 
there is no evident mechanism for this regulation. Motivated by several studies which proposed that cholesterol-which is a neutral lipid and one of the main components of the plasma membrane-may affect $\mathrm{Na}^{+}$binding, ${ }^{17,18}$ we have studied in detail the underlying mechanisms determining $\mathrm{Ca}^{2+}$ and $\mathrm{Na}^{+}$ interactions at the membrane surface. We find that the number of bound calcium ions depends on the total membrane area, whereas the number of phospholipid head groups determines the number of bound sodium.

To unravel the mechanisms governing $\mathrm{Ca}^{2+}$ and $\mathrm{Na}^{+}$interactions at the membrane surface, we have characterized the properties of liposomal membranes using fluorescence measurements in membranes with and without cholesterol, at different temperatures, and in the presence of varying levels of $\mathrm{Na}^{+}$and $\mathrm{Ca}^{2+}$. Furthermore, we have performed molecular dynamics simulations to investigate how increasing cholesterol levels and temperature affect the binding of $\mathrm{Ca}^{2+}$ and $\mathrm{Na}^{+}$to the membrane. Remarkably, results from both of these methodologies show excellent agreement with each other, with the latter one providing a detailed insight into the microscopic origins of our experimental observations.

To compare the impacts of the binding of sodium and calcium ions on model membranes, we performed fluorescence measurements with the fluorophore Laurdan. Laurdan probes the hydration and packing around the lipid $s n-1$ carbonyl region. ${ }^{19}$ We have performed two sets of experiments-time-dependent fluorescence shift (TDFS) and excitation generalized polarization $\left(\mathrm{GP}_{\mathrm{EX}}\right)$ - the latter serving as a reference methodology. TDFS presents several advantages with respect to $\mathrm{GP}_{\mathrm{EX}}$; while $\mathrm{GP}_{\mathrm{EX}}$ is a ratiometric phenomenological experiment that allows us to roughly distinguish between lyotropic lipid phases, ${ }^{20}$ TDFS allows the deconvolution of hydration (i.e. polarization) and mobility (i.e. packing) at the carbonyl region into two parameters-the emission shift $(\Delta \nu)$ and the relaxation time $\left(\tau_{\mathrm{r}}\right)^{21}$

No significant differences in the emission shifts $(\Delta \nu)$ of Laurdan were detected for the herein studied systems. This means that the probe does not detect any changes in membrane hydration, which we further discuss in Section S3.2 in the ESI. $\dagger$ The relaxation times $\left(\tau_{\mathrm{r}}\right)$ measured for Laurdan embedded in large unilamellar vesicles composed of either pure palmitoyloleoylphosphatidylcholine (POPC) or its 4:1 mixture with cholesterol (POPC/ $\mathrm{Chol}$ ) are shown in Fig. 1. The influences of sodium and calcium are studied at three different ion concentrations (0, $150 \mathrm{mM}$ and $1 \mathrm{M})$, all at $310 \mathrm{~K}$. Additional measurements at $288 \mathrm{~K}$ and $298 \mathrm{~K}$ provide similar trends (see Section S3.2, ESI $\dagger$ ).

As shown in Fig. 1, we observe significant changes in the relaxation time $\tau_{\mathrm{r}}$ as a function of cholesterol content and salt concentration. Further data measured at different temperatures are shown in Fig. S7 (ESI $\dagger$ ). Decreasing the temperature or increasing the cholesterol concentration increases the time required for the environment of Laurdan to relax. The former leads to a decrease in the area per lipid, whereas the latter results in a more ordered and rigid membrane. This means that in both cases the mobility in the carbonyl region-where Laurdan resides-decreases, which results in the slowdown of Laurdan rotational dynamics. A decrease in the temperature by $22 \mathrm{~K}$ has a larger effect than the addition of $20 \%$ cholesterol to the system (see Fig. S7, ESI $\dagger$ ). However, the addition

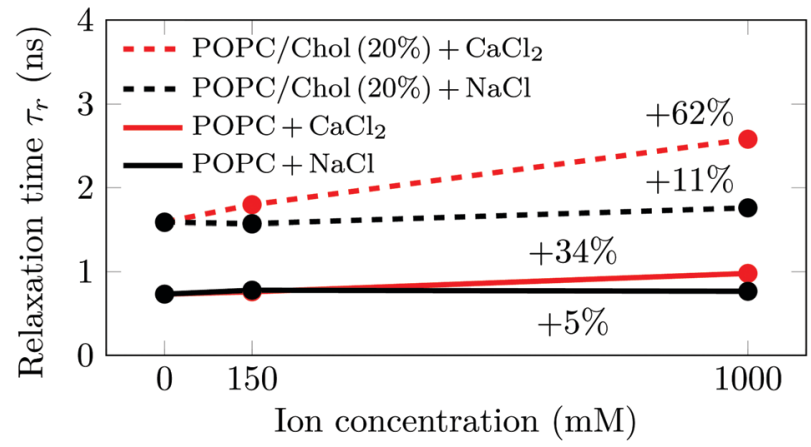

Fig. 1 Relaxation times from time-dependent fluorescence shift measurements at $310 \mathrm{~K}$. The percentage values represent the relative increase of Laurdan relaxation times upon the addition of $1 \mathrm{M}$ of salt. Although the methodology itself is quite precise (error of $\sim 0.05 \mathrm{~ns}$ ), we estimate the total error associated with the values for the relative increase to be $\sim 10$ percentage points, estimated by comparing values obtained using different fitting strategies.

of $\mathrm{Na}^{+}$at any investigated concentration does not have a significant effect on either POPC $\left(\Delta \tau_{\mathrm{r}}^{\text {Pure }}=5 \%\right)$ or POPC/Chol $\left(\Delta \tau_{\mathrm{r}}^{\text {Chol }}=11 \%\right)$ membranes, both values falling within the error estimate. This suggests that sodium cations do not significantly disturb the lipid surface structure. Therefore, the structural and dynamic properties of the membrane region where Laurdan resides remains unaltered.

In contrast, the addition of $\mathrm{Ca}^{2+}$ affects the measured relaxation times severely. This shows that calcium adsorption is sensed at the carbonyl level, while no direct probe-ion interactions are present (see Section S3.2, ESI $\dagger$ ). This is caused by the bridging of lipid molecules by calcium ions and the subsequent lateral membrane compression which results in the hindrance of the phospholipid mobility. ${ }^{11,22}$ Interestingly, it is clearly visible that the addition of cholesterol at $310 \mathrm{~K}$ not only increases the relaxation times, but also significantly amplifies the effects of calcium, i.e. from $\Delta \tau_{\mathrm{r}}^{\text {Pure }}=34 \%$ to $\Delta \tau_{\mathrm{r}}^{\mathrm{Chol}}=62 \%$. This amplified effect of calcium due to the presence of cholesterol is consistent with an increased number of $\mathrm{Ca}^{2+}$ per phospholipid adsorbed on the membrane. These additional ions cause further phospholipid bridging, lower mobility of the lipids around Laurdan, and consequently larger relaxation times.

Motivated by our unexpected finding that cholesterol modulates the binding of $\mathrm{Na}^{+}$and $\mathrm{Ca}^{2+}$ in different ways, and aiming to understand the molecular mechanisms behind this behavior, we simulated several phosphatidylcholine (PC) and PC/Chol membranes under various ionic environments (no ions, $130 \mathrm{mM}$ or $1 \mathrm{M}$ of $\mathrm{NaCl}$, or $450 \mathrm{mM}$ of $\mathrm{CaCl}_{2}$, see Table S1, ESI $\dagger$ ). Upon the addition of cholesterol to a given PC membrane, the total membrane area is increased (see Section S5.1, ESI $\dagger$ ). However, due to the condensing effect of cholesterol, ${ }^{23}$ this increase is less than that in the case of ideal mixing. In systems with $\mathrm{NaCl}$, the total number of adsorbed sodium ions remains almost constant with the cholesterol content (see Fig. 2) leading to a decrease in the surface charge density of the bilayer. We also observe that at a higher $\mathrm{NaCl}$ concentration the number of bound cations increases. These findings indicate that the binding 


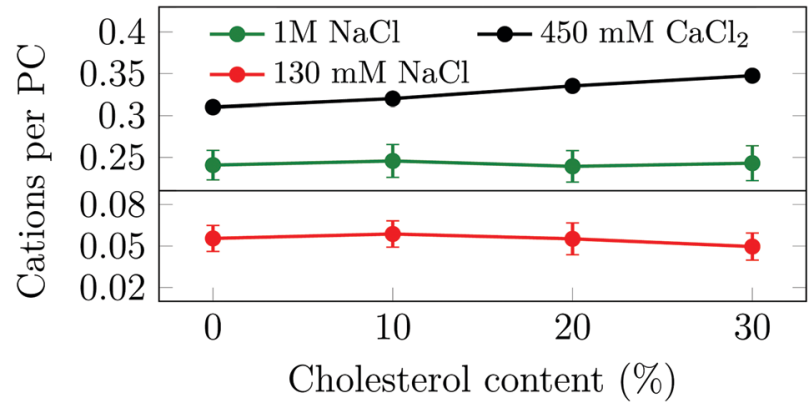

Fig. 2 Average number of bound cations per POPC head group as a function of cholesterol concentration. Error bars show std. dev.

of sodium depends solely on its bulk concentration and on the number of PC head groups available at the surface. Recent studies on the effect of cholesterol on the binding of sodium to PC membranes ${ }^{17,18}$ have reported seemingly contradicting results, which we found to be explainable by factors related to normalization (see Section S6, ESI $\dagger$ ).

Next, the same set of POPC bilayers with varying amounts of cholesterol were simulated in the presence of nominally $450 \mathrm{mM}$ of $\mathrm{CaCl}_{2}$. This amount of $\mathrm{CaCl}_{2}$ was selected so that after adsorption onto the membrane free cations would always remain in the aqueous phase for the present unit cell size. Unlike for $\mathrm{NaCl}$, the number of bound calcium ions increases as a function of the cholesterol content (see Fig. 2). The observed trends of the effect of cholesterol on both sodium and calcium binding are shared with nuances across several lipid and ion force fields (see Section S5.2, ESI $\dagger$ ).

Salt and cholesterol can disturb the lateral packing of the bilayer. The tendency of sodium and calcium to adsorb to bilayers is plotted as a function of area per PC in Fig. 3. We observe two distinct trends for $\mathrm{Na}^{+}$and $\mathrm{Ca}^{2+}$. The number of bound $\mathrm{Na}^{+}$per PC is constant regardless of the area per lipid (Fig. 3A). The independence of ion adsorption on the total membrane area results in variations of surface charge densities in the range of $\sim 0.07-0.12 \mathrm{e} \mathrm{nm}^{-2}$ in the simulated systems (Fig. 3B). To summarize, $\mathrm{Na}^{+}-$membrane interaction is mostly governed by the total number of PC head groups present. In the case of calcium, however, the number of adsorbed cations per PC depends roughly linearly on the available surface area (Fig. 3A), producing an approximately constant surface charge density of $\sim 1.06 \mathrm{e} \mathrm{nm}^{-2}$ in the present simulations (Fig. 3B). Notably, even a dipalmitoylphosphatidylcholine (DPPC) bilayer in the gel phase follows the same observed trend for both cations. The effects of increased temperature on both sodium and calcium adsorptions are similar but somewhat more complex (see Section S4.2, ESI $\dagger$ ).

The differences between $\mathrm{Na}^{+}$and $\mathrm{Ca}^{2+}$ reported by the present experiments and simulations are explained by the different natures of their binding to the membrane. Although sodium has a low binding energy, calcium binding is nearly irreversible. Our simulations also suggest that while sodium shows rapid exchange between the membrane surface and the bulk solvent, the concentration of calcium saturates at the surface relatively

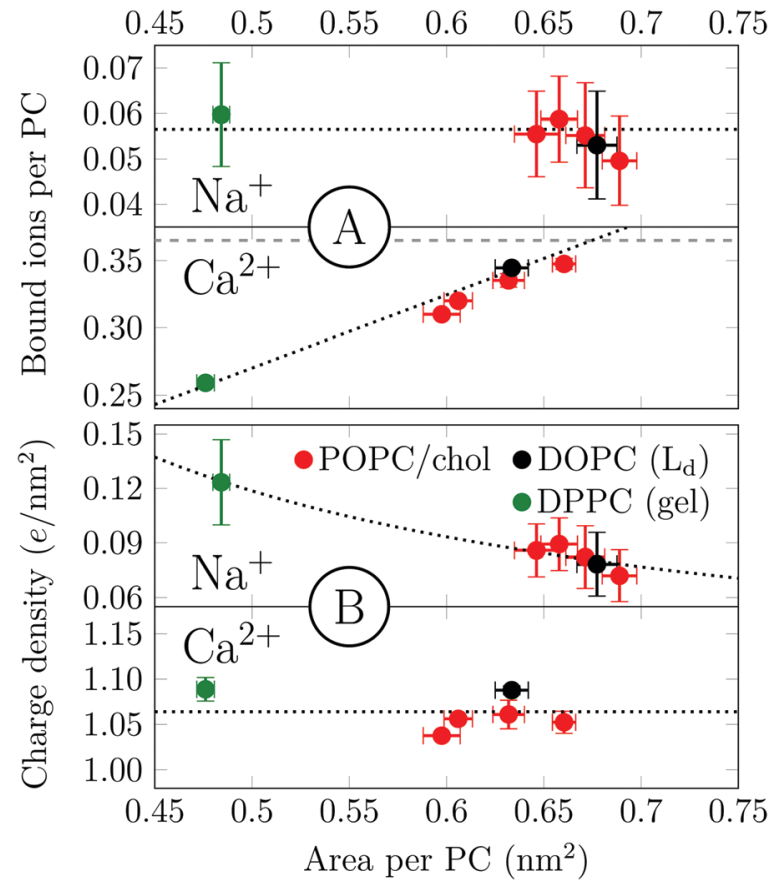

Fig. 3 (A) The dependence of cation adsorption $(130 \mathrm{mM}$ of $\mathrm{NaCl}$ or $450 \mathrm{mM}$ of $\mathrm{CaCl}_{2}$ ) on the area per PC. (B) The dependence of surface charge density on the area per PC in membranes. Red dots are POPC with $0,10,20$, or $30 \%$ cholesterol at $310 \mathrm{~K}$ (smallest to highest area). Black dot is DOPC at $310 \mathrm{~K}$. Green dot is DPPC at $295 \mathrm{~K}$. Dashed lines show fits to the data; see text for details. Error bars show std. dev.

slowly (see Section S5.3, ESI $\dagger$ ), and desorption events are rare. In both cases, the amount of phosphatidylcholine lipids determines the number of possible binding sites; however, a real competition for them only exists for $\mathrm{Ca}^{2+}$. With $\mathrm{Na}^{+}$, most binding sites are essentially free or only transiently occupied. Under these conditions, only the bulk $\mathrm{Na}^{+}$density and the rather constant surface PC density determine the number of surface bound $\mathrm{Na}^{+}$. This finding indicates that the $\mathrm{Na}^{+}$concentration would be enhanced in ordered membrane domains with higher density of PC head groups, i.e. binding sites. This is indeed observed in heterogeneous ternary systems ${ }^{24}$ (see Section S4.1, ESI $\dagger$ ).

In contrast to sodium, the strong binding of calcium results in a practically complete coverage of the membrane surface area at the relatively high calcium concentrations employed here. In our simulations, we find that each calcium is coordinated on average to three PC phosphate groups (Section S5.4, ESI $\dagger$ ). This strong coordination at the head group level slows down lipid rotation (Section S4.3, ESI $\dagger$ ) and self-diffusion (Section S5.1, ESI $\dagger$ ).

An increase in cholesterol concentration leads to higher calcium adsorption even though cholesterol itself neither interacts with the ions nor does it affect the penetration depth. Cholesterol thus does not seem to take part in the calcium coordination and the number of coordinated phosphates is unaffected by the presence of cholesterol. These results indicate that cholesterol simply allows more calcium adsorption by increasing the membrane area maintaining a fairly constant critical surface charge density. This unspecific role is emphasized 


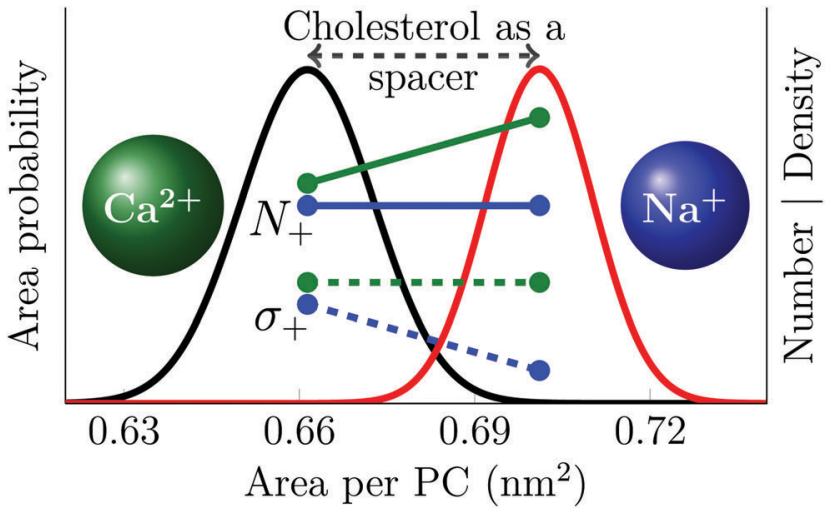

Fig. 4 Schematic picture describing the effect of cholesterol on cation adsorption. Black and red curves show the distribution of the area per phospholipid in POPC membranes with $0 \%$ and 30\% cholesterol, respectively. Green and blue lines show data for the effect of cholesterol-induced area increase on calcium and sodium adsorption respectively; solid lines show the number of adsorbed ions $\left(N_{+}\right)$, while the dashed lines show the resulting surface charge density $\left(\sigma_{+}\right)$. The number of bound calcium ions is proportional to the total area, resulting in a constant surface charge density. The number of sodium ions, on the other hand, is proportional to the number of PC head groups, resulting in a surface charge density that is proportional to the area per PC lipid. Note that this figure depicts trends; the data are properly shown in Fig. 2 and 3.

by the fact that other ways of affecting the membrane area (e.g. by increasing lipid unsaturation or by taking the membrane to a gel phase) lead to similar observations. This suggests that in this case the charge-charge repulsion between $\mathrm{Ca}^{2+}$ ions is the limiting factor of adsorption. A detailed explanation of all these observations can be found in the $\mathrm{ESI} \dagger$ (Section S5).

In conclusion, we have performed fluorescence measurements in POPC membranes with increasing cholesterol and sodium or calcium concentrations and complemented the results by simulating analogous systems using different lipid and ion force fields with the aim to unravel the effect of cholesterol on $\mathrm{Na}^{+}$and $\mathrm{Ca}^{2+}$ cation adsorption onto lipid bilayers. We find that the interaction of $\mathrm{Na}^{+}$ with the membrane depends on the total number of PC head groups present, which determines the number of binding sites. In contrast, the level of adsorbed calcium is determined primarily by the accessible surface area of the membrane.

This significantly different behavior of the two cations results from the weak and transient sodium binding with the lipid head groups that contrasts with the stronger calcium adsorption that is capable of inducing the clustering of phosphatidylcholine head groups. There is quantitative agreement between simulations and experiments concerning these observations, which are graphically summarized in Fig. 4.

The present findings indicate that a cell-which can control its global and local membrane lipid compositions-may use this ability to selectively modulate the membrane interaction with $\mathrm{Na}^{+}$and $\mathrm{Ca}^{2+}$ by inducing lateral heterogeneity (regions of varying lipid packing) or controlling its volume. Taking into consideration the important role of these two cations in the regulation of cellular functions, the mechanisms explained here could be exploited by the cell and may constitute a hitherto unrecognized regulation mechanism. The present findings also help us to understand how cations interact with cholesterol-rich ordered regions in heterogeneous membranes. This information may prove crucial for understanding the role of ions in modulating the adsorption of charged molecules onto the membrane surface.

We thank CSC - IT Center for Science (Espoo, Finland) for computing resources. HMS and MJ thank the Academy of Finland (Centre of Excellence program) and the European Research Council (Advanced Grant CROWDED-PRO-LIPIDS) for financial support. PJun acknowledges support from the Czech Science Foundation (grant no. 16-01074S) and the Academy of Finland via the FiDiPro program. AM, PJur and MH acknowledge the Czech Science Foundation (grant no. 17-03160S) and the Czech Academy of Sciences for the Praemium Academiae award.

\section{References}

1 T. J. Jentsch, Nat. Rev. Mol. Cell Biol., 2016, 17, 293-307.

2 O. Sten-Knudsen, Biological Membranes: Theory of Transport, Potentials and Electric Impulses, Cambridge University Press, New York, 2002.

3 O. S. Andersen, Encyclopedia of Metalloproteins, Springer Science + Business Media, 2013, pp. 580-587.

4 K. Hu, J. Carroll, S. Fedorovich, C. Rickman, A. Sukhodub and B. Davletov, Nature, 2002, 415, 646-650.

5 P. K. Tarafdar, H. Chakraborty, S. M. Dennison and B. R. Lentz, Biophys. J., 2012, 103, 1880-1889.

6 M. J. Berridge, M. D. Bootman and H. L. Roderick, Nat. Rev. Mol. Cell Biol., 2003, 4, 517-529.

7 S. Orrenius, B. Zhivotovsky and P. Nicotera, Nat. Rev. Mol. Cell Biol., 2003, 4, 552-565.

8 O. Szekely, A. Steiner, P. Szekely, E. Amit, R. Asor, C. Tamburu and U. Raviv, Langmuir, 2011, 27, 7419-7438.

9 A. Y. Antipina and A. A. Gurtovenko, J. Phys. Chem. B, 2015, 119, 6638-6645.

10 J. J. McManus, J. O. Rädler and K. A. Dawson, J. Phys. Chem. B, 2003, 107, 9869-9875.

11 P. Jurkiewicz, L. Cwiklik, A. Vojtšková, P. Jungwirth and M. Hof, Biochim. Biophys. Acta, Biomembr., 2012, 1818, 609-616.

12 A. Melcrová, S. Pokorna, S. Pullanchery, M. Kohagen, P. Jurkiewicz, M. Hof, P. Jungwirth, P. S. Cremer and L. Cwiklik, Sci. Rep., 2016, 6, 38035.

13 A. Catte, M. Girych, M. Javanainen, C. Loison, J. Melcr, M. S. Miettinen, L. Monticelli, J. Määttä, V. S. Oganesyan, O. S. Ollila, J. Tynkkynen and S. Vilov, Phys. Chem. Chem. Phys., 2016, 18, 32560-32569.

14 M. N. Jones, Adv. Colloid Interface Sci., 1995, 54, 93-128.

15 J. Melcr, D. Bonhenry, Š. Timr and P. Jungwirth, J. Chem. Theory Comput., 2016, 12, 2418-2425.

16 Y. Saheki, X. Bian, C. M. Schauder, Y. Sawaki, M. A. Surma, C. Klose, F. Pincet, K. M. Reinisch and P. D. Camilli, Nat. Cell Biol., 2016, 18, 504-515.

17 J. Yang, M. Bonomi, C. Calero and J. Martí, Phys. Chem. Chem. Phys., 2016, 18, 9036-9041.

18 A. Magarkar, V. Dhawan, P. Kallinteri, T. Viitala, M. Elmowafy, T. Róg and A. Bunker, Sci. Rep., 2014, 4, 1-5.

19 P. Jurkiewicz, L. Cwiklik, P. Jungwirth and M. Hof, Biochimie, 2012, 94, 26-32.

20 T. Parasassi, G. De Stasio, A. d'Ubaldo and E. Gratton, Biophys. J., 1990, 57, 1179-1186.

21 M. Amaro, R. Sachl, P. Jurkiewicz, A. Coutinho, M. Prieto and M. Hof, Biophys. J., 2014, 107, 2751-2760.

22 R. A. Böckmann and H. Grubmüller, Angew. Chem., Int. Ed., 2004, 43, 1021-1024.

23 T. Róg, M. Pasenkiewicz-Gierula, I. Vattulainen and M. Karttunen, Biochim. Biophys. Acta, Biomembr., 2009, 1788, 97-121.

24 M. Javanainen, H. Martinez-Seara and I. Vattulainen, Sci. Rep., 2017, 7, 1143 . 